\title{
A framework for understanding quality of life in individuals without capacity
}

\author{
Jeff Round • Elizabeth L. Sampson • \\ Louise Jones
}

Accepted: 1 August 2013/Published online: 22 August 2013

(C) Springer Science+Business Media Dordrecht 2013

\begin{abstract}
Purpose The wide range of tools and methods developed for measuring and valuing health-related quality of life for use in economic evaluations are appropriate for use in most populations. However, for certain populations, specific tools may need to be developed to reflect the particular needs of that population. Patients without capacity-particularly patients with severe dementia-are such a population. At present, the tools available to economists for measuring and valuing quality of life in these patients lack validity. Here, we seek to understand the framework within which common instruments have been developed, critique these instruments with respect to patients with severely restricted capacity and to develop a new way of thinking about how to value healthrelated quality of life in such patients.

Method In this essay, we describe and critique the conceptual framework by which common instruments used for measuring and valuing quality of life have been developed. Results We show that current common instruments used for measuring and valuing quality of life in general populations are not appropriate for populations with severely restricted capacity.
\end{abstract}

J. Round $(\bowtie) \cdot$ E. L. Sampson · L. Jones

Marie Curie Palliative Care Research Unit, Mental Health

Sciences Unit, University College London, Charles Bell House,

67-73 Riding House St, London W1W 7EJ, UK

e-mail: j.round@ucl.ac.uk

E. L. Sampson

e-mail: e.sampson@ucl.ac.uk

L. Jones

e-mail: caroline.jones@ucl.ac.uk

E. L. Sampson

Barnet Enfield and Haringey Mental Health Trust, St Ann's

Hospital, St Ann's Road, London N15 3TF, UK
Conclusions We propose a new framework for thinking about quality of life in this population, based on notions of observable person-centred outcomes and utility derived from processes of care.

Keywords Quality of life - Dementia: capacity . End of life care $\cdot$ Resource allocation

\section{Introduction}

Economic evaluation is an established tool for decision makers and commissioners in the allocation of scarce healthcare resources. For the results of economic evaluations to be considered valid, the methods and measurement instruments used must be appropriate to the patient population being studied. In most cases, the established methods and tools of the health economist [1-3] can be applied with little need for adaptation to account for the specific patient population. There are, however, some populations and fields of care where standard methodologies often do not satisfy the needs of analysts and decision makers. These shortcomings are particularly evident in the measurement of health-related quality of life. Areas where it has been argued that current quality of life measurement tools are not adequate include mental health, public health, social care and patients nearing death [4].

In this essay, we consider a group of people where current methods for the measurement of quality of life do not reflect their needs or the needs of those tasked by society with allocating resources. This group is comprised of people without the capacity to take part in the assessment of their own health. In particular, we focus on people with advanced dementia. Dementia is a neurodegenerative disease which, in its advanced stages, leads to profound 
global cognitive and functional deficits (unable to recognise members of their family, can speak only a few words, unable to ambulate independently, care for themselves and are doubly incontinent) [5, 6]. People at this stage of dementia typically have little ability to speak or communicate and will require extensive help in the activities of daily living. We argue that current models for understanding and measuring quality of life lack descriptive validity (that is, content, face and construct validity) for this population and that their use is therefore inappropriate for allocating resources.

To make this argument, we first provide an overview of the dominant framework in economic evaluation for measuring and valuing health-related quality of life and methods for the estimation of quality-adjusted life years. We then consider and appraise critically the current frameworks for assessing health-related quality of life currently practised, including functioning (performance), the capabilities approach and the notion of subjective wellbeing (SWB). Then, we show how these frameworks lack validity in a population with extremely limited cognitive and physical capacity with respect to their ability to evaluate their own health or well-being; people with advanced dementia are used as an exemplar population but such cognitive deficit may be present in people with other health conditions. We finish by proposing an alternative conceptual framework for developing an instrument to measure quality of life in this group that is compatible with current standard evaluative frameworks such as that used by UK National Institute for Clinical Excellence (NICE).

\section{Economic evaluation and the QALY}

The role of economic evaluation in healthcare is to provide information for decision makers on the relative costs and benefits of competing demands for resources. A particular approach to economic evaluation-cost-utility analysis (CUA) - has become a key analytical tool for health economists. Within cost-utility analysis, benefit from treatment is measured in terms of quality-adjusted life years (QALYs). The QALY is a metric that combines information on both the duration and quality of life following treatment. The use of the QALY allows different healthcare interventions for the same condition to be compared using a single measure of benefit. More broadly, it also allows for interventions across myriad patient groups to be compared. As a result, decision makers are able to make judgements on which basket of treatments to purchase to meet a specified goal-for example, an improvement in the health of a population [7].

It has been argued that under the existing CUA framework, the QALY is not an appropriate measure of benefit for certain groups, such as those nearing the end of their lives or those without the potential for improvement in health [8-10]. At the core of these arguments are the notions that certain populations require either (1) care that is unlikely to lead to improvements in length of life or (2) that the QALY framework is necessarily biased against such patients. These arguments have been addressed in detail elsewhere $[11,12]$, and it is our view that the QALY can be meaningfully applied to such groups, given the right set of methodological tools to derive estimates of health state utility values. The current difficulty lies in the absence of such a set of tools. Using current instruments for measuring and valuing outcomes would mean many, if not most, interventions for people without cognitive capacity and with a poor prognosis would not be considered costeffective [9]. Yet, it would be unethical and immoral not to provide at least a basic level of care and comfort to individuals in need. The problem that remains could be described as the 'caring problem'-how to measure and value treatment where the aim is to provide care for patients where there is little expectation of an improvement in the state of health or length of life, such as people with advanced dementia?

\section{Conceptual frameworks in quality of life measurement}

Instrument validity and people without cognitive capacity

Validity is the idea that an instrument is measuring what it is claimed to be measuring [13]. There are numerous ways of assessing the validity of an instrument [13], but the immediate concern is whether current preference-based instruments exhibit content and face validity in patients without capacity. These facets of validity reflect whether the items are appropriate to the population being studied [14]. Content validity is concerned with the degree to which an instrument covers the dimensions of health relevant to the population and the degree to which it is sensitive to changes in health status [14]. Face validity is a less formal method of assessment and considers whether the items are sensible and appropriate for the study population [14]. We argue that current instruments, developed under the three existing conceptual frameworks (described below), lack content and face validity for people with severe dementia or for other populations lacking cognitive capacity.

Existing conceptual frameworks

There are no universally accepted labels to describe the different conceptual frameworks under which existing 
preference-based quality of life instruments have been developed. However, it is useful to differentiate between three particular concepts that have influenced instrument development. The three categories are not necessarily mutually exclusive; some instruments may include questions that cover more than one category. Nevertheless, it is still useful to understand the dominant conceptual frameworks that have underpinned the development of current instruments.

\section{Functioning}

The first class of instruments described here are those that focus on functioning or performance, such as the EQ-5D, the SF-6D and the Health Utilities Index (HUI), three of the most commonly used instruments [14]. These include a series of questions about domains accepted as nominally health related — such as pain or mobility — and people are asked to evaluate how an illness or a treatment impacts their functioning in each of those domains. This functional approach measures how successfully they feel that they perform against a set of pre-specified criteria believed to be important in defining their quality of life. In the case of the person with severe dementia, who lacks even the most basic ability in activities of daily living, and is unable to respond, such an instrument lacks content and face validity. Not only will people with advanced dementia receive the lowest possible score on domains such as mobility, selfcare or usual activities, the progressive nature of the disease means that there are no interventions currently available that could improve that score. Additionally, more subjective measures of functioning - such as freedom from pain, or feelings of anxiety and depression-may not be answered directly by the individual. This matters, as research shows that responses provided by proxy often differ from direct reporting when completing outcome measures [15-17] and that responses to less objective domains (e.g. mental health) may vary more than responses to more objective domains (e.g. physical functioning) in older adults [18]. In advanced dementia, the accuracy of proxy assessments is further compromised by the level of skill required in detecting symptoms such as pain [19].

\section{Capability}

Recently, two alternative frameworks for conceptualising quality of life have been proposed. The first of these is based on the concept of capabilities [20] and is exemplified by the ICECAP [4] and ICEPOP instruments [21]. The capability approach seeks to define quality of life in terms of what people can do and be in their life [4]. This differs from the functional approach, as the emphasis is placed on whether or not a person could achieve an objective should they so choose. The aim, as described by Al-Janabi and colleagues, is to '. . avoid imposing a particular idea of what a good life constitutes and to reflect the importance of freedom to choose' [4]. This principle is closely related to the notion of autonomy - that is, the individual's right to partake in informed decisions about their life, their health and their care. This autonomy is absent in persons with severe dementia, and related domains are not relevant to the population group.

To contrast this approach with the functional approach, where the EQ-5D asks about mobility or depression, the ICECAP-A asks questions about whether an individual could be independent if they chose, or whether they are able to experience enjoyment or pleasure. Again, the domains being measured by capability-based instruments do not exhibit content or face validity in people with severe dementia-they have little independence, nor will they ever again have the autonomy be able to choose what they wish to do. Where a third party can make an assessment, the person with dementia will often score low on the scale without scope for an improvement. For example, the ICECAP-SCM includes a domain about having a say in the care they receive-this is, simply not possible for individuals with severe cognitive impairment, and any proxy response would have to be at the lowest possible level for that item. And as with the functional scales, where questions are more subjective (e.g. ability to feel settled and secure), proxy responses cannot be considered as reliable [18].

\section{Subjective well-being}

The assessment of SWB is based on a different methodological as well as conceptual approach. Dolan, Layard and Metcalfe [22], proponents of the adoption of subjective measures of well-being into public policy development and analysis, use the definition given by the Stiglitz Commission (which reported on the measurement of economic performance and social progress) [23]. In their report, the Commission defined SWB as about providing a '...cognitive evaluation of one's life'. Such evaluations can encompass happiness, satisfaction or other positive emotions, as well as negative emotions such as pain or worry. Dolan et al. [22] highlight that a key strength of this approach is that people are able to decide for themselves whether their life is a good one or not and that the questions used are conceptually simple. The application of SWB to economic evaluation is at an embryonic stage, and it is not clear whether it could (or should) supplant current methods. SWB has at its core the notion that the individual is the best judge of their wellbeing, yet it is clear that the method is inappropriate for people without the cognitive capacity to evaluate their life. It would therefore be left to 
an external observer to provide the assessment, and there is evidence that important discrepancies can exist between observer and patient assessments of quality of life [18].

Dementia-specific instruments

In using severe dementia as an exemplar population, we give specific consideration to some of the key instruments developed specifically for use in this population. These include the DEMQOL [24], QUALID [25] (Quality of Life in Late-Stage Dementia) and Quality of Life-Alzheimer's Disease [26] (QOL-AD) instruments. At present, none of these has a corresponding set of preferences from which utility values could be estimated for use in economic evaluation, though such a value set could be developed in future. Alternatively, these non-preference-based instruments could be 'mapped' to existing preference-based instruments - an increasingly common approach in health economic analyses where validated preference-based instruments are not available though the usefulness of socalled mapping techniques has been challenged on grounds that predictive performance is variable [27] and that results may not be generalisable to populations other than that in which the mapping algorithm is derived [28].

Consideration of the DemQoL and QOL-AD (Table 1) suggests they have been developed primarily within a functional or performance conceptual framework, reflecting the inclusion of domains focused on physical or mental health and relationships with others. In addition, some domains reflect a capabilities approach, such as an ability to do chores or to have fun, or whether people have enough money. The QOL-AD is reported as having been developed with reference to four domains identified by Lawton [29] as being important to older adults [26]: behavioural competence, environment, psychological wellbeing and perceived quality of life. As argued above, it is our view that functional- or capabilities-based instruments will necessarily lack content and face validity for use with patients with advanced dementia or other severe cognitive impairment.
In contrast, the QUALID does not fit comfortably within any of the three categorisations-functioning, capability or SWB-outlined in the preceding section. Instead, this instrument focuses on domains that can be observed and reported by a third party such as a healthcare provider or someone who has a personal relationship with the person being assessed. These domains cover objective reporting of observer assessment of the emotional state of the person as well as signs of pain, agitation and mental distress. While some domains may be difficult for proxy respondents to answer accurately (e.g. those focusing on the emotional wellbeing of the individual, presence or absence of obvious distress), others are more subjective and appear to be highly relevant (e.g. observed behaviours such as aggression, apparent physical comfort). This instrument exhibits the greatest degree of face validity for use in a population of people with severe dementia when compared with the DEMQOL and QOL-AD. However, the QUALID is not a good candidate for further development as a preferencebased instrument; there are too many items and response levels, generating an unmanageable number of unique health states (nearly 50 million) for reliable valuation [14, 30]. However, despite these limitations, the QUALID highlights important criteria that may be applied in the development of a preference-based measure for use in patients without capacity. These lessons are considered in the following section.

\section{An alternative conceptual framework for instrument development}

We have argued that the current dominant conceptual frameworks for preference-based instrument development are inadequate for use in people with severe dementia. In rejecting the existing approaches, the onus thus falls on us to offer a replacement conceptual framework. In this section, we provide what might be described as the scaffolding from which a new concept of thinking about quality of life
Table 1 Overview of selected dementia-specific quality of life instruments

\begin{tabular}{lll}
\hline Instrument & Domains & Framework \\
\hline DEMQOL & $\begin{array}{l}\text { Feelings (primarily focused on mental health) } \\
\text { Memory } \\
\end{array}$ & Functioning/capabilities \\
& Overyday Life & \\
QOL-AD & $\begin{array}{l}\text { Behavioural competence Environment } \\
\text { Psychological wellbeing }\end{array}$ & Functioning/capabilities \\
& $\begin{array}{l}\text { Perceived quality of life } \\
\text { Qental health }\end{array}$ & \\
& Physical health & Functioning/objective assessment \\
\hline
\end{tabular}


may be constructed. We refer to this as a capacity-adapted approach.

We propose that the foundations of this new approach are based on two fundamental considerations. First, that domains and items relating directly to the person should be based on criteria that can be assessed objectively. Second, that the process of care very much matters in this population, indeed more than it might in others. Therefore, the notion of process-of-care utility is highly relevant. Finally, in addition to the underlying conceptual foundation, any future instrument should be developed so that it is suitable for valuation in a manner that will allow QALYs to be calculated and compared with values derived from alternative instruments. Our proposed approach is summarised in Fig. 1.

\section{Objective assessment}

People with severe dementia are unable to complete a selfcompletion questionnaire; therefore, any assessment of their health state must be made by a third party. The evidence shows that concurrence between proxy and person responses is better for objective domains than it is for subjective domains [16-18]. Those domains that have been shown to have high concurrence between person and proxy respondents should be considered for inclusion; it is likely that these will be objective rather than subjective.

A brief overview of the literature on response agreement suggests that a number of objective domains could be considered. A study of people with mild-to-moderate Alzheimer's disease, in whom cognitive impairment is less, found response agreement between people and proxies was best (though still only moderate) for physical health and disability [31]. Specific items that may be important in people with severe dementia may include markers of general comfort, pain control, control of agitation and ensuring adequate hydration and feeding and their response to pleasant stimuli such as touch, smell and sound [32, 33].

Process of care and process utility

In addition to items offering an objective assessment of health status, we propose that the process of care is important in this population. Process-of-care utility is not typically considered within preference-based measures of health-related quality of life. Instead, utility from a treatment is typically considered to be derived from the results of a process rather than the process itself [34]. By contrast, a measure of process-of-care utility would give weight to any utility derived from the process-of-care itself, separate

\begin{tabular}{|c|c|c|}
\hline 范 & $\begin{array}{l}\qquad \text { Existing approaches } \\
\text { - Primarily assess functioning or capabilities of } \\
\text { individuals } \\
\text { - Rely on subjective evaluation of domains or } \\
\text { attributes }\end{array}$ & $\begin{array}{l}\text { Capacity adapted approach } \\
\text { - Some individuals do not have capacity to } \\
\text { complete instruments } \\
\text { - Use of proxy assessment of objective criteria }\end{array}$ \\
\hline 莺 & $\begin{array}{l}\text { Existing approaches focus on: } \\
\text { subjective assessment of health states (subjective } \\
\text { well-being) } \\
\text { - assessing either functioning (EQ-5D, SF-6D) or } \\
\text { capabilities (ICECAP) or a combination (DEMQOL) } \\
\text { In those without capacity, existing instruments } \\
\text { - lack descriptive validity } \\
\text { - require completion by proxy, though unreliable } \\
\text { for subjective attributes } \\
\text { - allow no/minimal scope for improvementin } \\
\text { scores/utility values }\end{array}$ & $\begin{array}{l}\text { Capacity can be: } \\
\text { - fluctuating (drugs, end of life, brain tumours) } \\
\text { - deteriorating (progressive neurological diseases) } \\
\text { - reversible (delirium, infection) } \\
\text { - irreversibly lost (dementia) } \\
\text { Care processes matter to individuals and the } \\
\text { population (caring externalities) } \\
\text { A capacity adapted approach focuses on attributes } \\
\text { and processes of care thatcan be assessed objectively } \\
\text { Allows for valuation of caring, not just health status }\end{array}$ \\
\hline 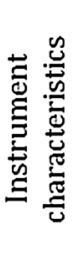 & $\begin{array}{l}\text { Subjective health domains } \\
\text { - General quality of life } \\
\text { - Feelings } \\
\text { - } \text { Dignity } \\
\text { - Enjoyment } \\
\text { - } \text { Confididence } \\
\text { - Quality of relationships } \\
\text { - Depression/Anxiety }\end{array}$ & $\begin{array}{r}\text { Objective elements of care } \\
\text { Pain assessment and control } \\
\text { - Place of care preferences } \\
\text { - Symptom management } \\
\text { (eg nausea, dyspnoea) } \\
\text { - Advance care planning } \\
\text { Attention to spiritual needs } \\
\text { Involvement of close persons } \\
\text { in care decisions }\end{array}$ \\
\hline
\end{tabular}

\section{Instrument}

- Measures characteristics relevant to population of interest

- Suitable for valuation and use in economic evaluation

Fig. 1 The capacity-adapted approach for measuring quality of life in patients with severe dementia 
from the outcome. In people whose health status is unlikely to change as a result of treatment, this process-of-care may increase in importance.

The appeal of considering process-of-care in this population is that people (or society) may derive value from the actual processes of providing care, as opposed to achieving some desired level of health or improved health state. As has been described, there is little scope for improvement in the state of health for people with severe dementia. Giving greater weight to the process by which care is delivered may lead to a more accurate assessment of the quality of life experienced by the person receiving that care. In this respect, consideration of process-of-care acts as a proxy measure for a particular health state where that health state cannot be measured directly. It may also reflect a societal view that values providing care to patients even where the scope for health improvement is limited or nonexistent.

As part of a programme of work exploring interventions for advanced dementia [35], we have undertaken a series of interviews with carers and healthcare professionals working in this field. We will use the results of this study to help determine what domains and processes of care are considered important. A systematic review undertaken as part of this research has identified a number of elements that may be of interest, such as pain management, adequate feeding, comfort and care planning. These items are presented for illustrative purposes, as any instrument development would require a dedicated and robust research plan. What they do show is that such research is feasible and therefore we argue that it should be undertaken.

\section{Valuing process-of-care utility}

To develop an instrument that measures health-related quality of life is in itself an arduous task. To develop such an instrument that can then be used to estimate utility while accounting for the process-of-care is more difficult and time-consuming. We now introduce a further difficulty in that we believe it is necessary to incorporate process-ofcare utility explicitly within the instrument, alongside health states. This is made challenging due to the risk of double counting utility gains that may accrue through both the valuation of health states and the valuation of processof-care in a single instrument. It is also the case that gains owing to processes of care may be highly transitory in nature, occurring briefly on each occasion and not necessarily frequently repeated. To address this, we consider the application of Multi-Attribute Utility (MAU) theory, which we believe can be used to overcome each of these difficulties.

The MAU model is an established, theoretically grounded approach to economic decision making that allows the combination of multidimensional measures of outcome into a single outcome [36]. Key to the development of the MAU instrument is that the individual measures of outcome are measurable by a common standard. This approach has been used successfully to generate utility values for the different versions of the HUI, one of the functioning-based instruments discussed above [14]. We contend that the development of an instrument that satisfies the criteria of MAU theory is possible. Using MAU models to value health-related quality of life requires that each item or domain contained in the descriptive system of the instrument is first measured individually. A multiplicative or multi-linear model can then be used to combine this information, accounting for interactions between terms and thus removing the risk of double counting of health gains.

\section{Conclusion}

Conceptually, measuring quality of life is straightforward-find out what matters to people and then ask them about it; in practice, this is much more difficult. A broad and detailed literature has evolved to ensure that instruments are developed according to best research practice and to improve the prospects of instruments displaying the necessary characteristics of validity and reliability. Any new instrument development should follow as closely as possible this best practice. However, we do believe that any new instrument should demonstrate certain characteristics which should be borne in mind during development.

Firstly, any new instrument should be suitable for use in economic evaluation. As such, it should be designed with the intention of obtaining a value set of preferences associated with the states it defines. The most robust instruments typically exhibit a certain set of characteristics; for example, they should contain a maximum of nine domains, each domain should contain ordinal responses, and the questionnaire should be written in plain and clear language. Secondly, we argue that the set of health states should be valued according to the preferences of the general population. Although there is no universal agreement about which population should provide health state values, there are two strong arguments in favour of using population values. The normative argument is that as the general population bears the burden of paying for care, they should be given a say in what care is provided [40]; additionally, as we cannot identify the patient groups that bear the burden of the opportunity cost, it would be inequitable to allow specific patient groups to determine the value placed on specific treatment [41]. These arguments are addressed at greater length elsewhere [11, 42] More prosaically, this is the approach favoured by NICE in their guide to 
technology appraisals, and a novel instrument would have an increased likelihood of gaining acceptance in research settings if it were developed according to the currently applied methodological guidance.

A further complicated situation arises in populations in which individuals may suffer fluctuating capacity for a range of reasons and with variable prognoses, for example delirium. We do not attempt to address problems associated with these conditions in this discussion paper, but further instrument development would need to consider such circumstances. Finally, while we have focused here on people with severe dementia, any novel instrument would benefit from being suitable for use in people who lack cognitive capacity for any reason, provided that a suitable set of domains could be identified. This would improve the ability to compare treatments in respect of resource allocation decision making.

To conclude, the instruments currently widely used to measure health-related quality of life for economic evaluation are not suitable for use in populations without the ability or capacity to respond. We have shown in this essay why we believe this and why there is a need for a new instrument that assesses objective measures of outcome as well as incorporating benefits to be gained through the processes of providing care. As we have argued, instruments based on definitions of health status that focus on either functioning or capacity are not suitable-such approaches do not reflect the needs of, or care provided to, people with severe dementia or those who otherwise lack capacity. Such instruments lack face and content validity. The development of a novel instrument, reflecting the needs of this group, would lead to a more efficient allocation of scarce resources while also improving the quality of care received by people with impaired capacity.

Acknowledgments All author's posts are supported by Marie Curie Cancer Care core grant funding to the Marie Curie Palliative Care Research Unit, Department of Mental Health Sciences, University College London (Grant number MCCC-FCO-11-U). The funders had no role in the conception or preparation of, or decision to submit, this manuscript.

Conflict of interest No authors have a conflict of interest to report.

\section{References}

1. National Institute for Health and Clinical Excellence (2008) Guide to the methods of technology appraisal; National Institute for Health and Clinical Excellence.

2. Briggs, Andrew, Calxton, Karl, \& Sculpher, Mark. (1). Decision Modelling for Health Economic Evaluation. Oxford: Oxford University Press.

3. Drummond, Michael F. (2005). MJSGWTBJOaGLS: Methods for the Economic Evaluation of Health Care Programmes (Vol. 3). Oxford: Oxford University Press.
4. Al-Janabi, H., Flynn, T. N., \& Coast, J. (2012). Development of a self-report measure of capability wellbeing for adults: the ICECAP-A. Quality of Life Research, 21, 167-176.

5. Sclan, S. G., \& Reisberg, B. (1992). Functional assessment staging (FAST) in Alzheimer's disease: Reliability, validity, and ordinality. International Psychogeriatrics, 4(Suppl 1), 55-69.

6. Reisberg, B., Sclan, S. G., Franssen, E., Kluger, A., \& Ferris, S. (1994). Dementia staging in chronic care populations. Alzheimer Disease and Associated Disorders, 8(Suppl 1), S188-S205.

7. Drummond, M., Brixner, D., Gold, M., Kind, P., McGuire, A., \& Nord, E. (2009). Toward a consensus on the QALY. Value Health, 12(Suppl 1), S31-S35.

8. Normand, C. (2009). Measuring outcomes in palliative care: Limitations of QALYs and the road to PalYs. Journal of Pain and Symptom Management, 38, 27-31.

9. Chochinov, H. M. (2011). Death, time and the theory of relativity. Journal of Pain and Symptom Management, 42, 460-463.

10. Coast J, Lavender S. (2009). An exploration of the economics of end of life care; Health Economists Study Group, Winter 2009.

11. Round, J. (2012). Is a QALY still a QALY at the end of life? Journal of Health Economics, 31(3), 21-52.

12. Edlin, R., McCabe, C., Round, J., Wright, J., Claxton, K., Sculpher, M., \& Cookson, R. (2013). Understanding Harris' understanding of CEA: Is cost effective resource allocation undone? Journal of Health Services Research and Policy, 18(1), 34-39.

13. Streiner, D. L., \& Norman, G. R. (2003). Health Measurement Scales (Vol. 3). Oxford: Oxford University Press.

14. Brazier, J., Ratcliffe, J., Salomon, J., \& Tsuchiya, A. (2007). Measuring and Valuing Health Benefits for Economic Evaluation (ed) (Vol. 1). Oxford: Oxford University Press.

15. Selai, C., Trimble, M. R., Rosser, M. N., \& Harvey, R. J. (2012). Assessing quality of life in dementia: Preliminary psychometric testing of the Quality of Life Assessment Schedule (QOLAS). Neuropsychological Rehabilitation, 11, 219-243.

16. Magaziner, J., Simonsick, E. M., Kashner, T. M., \& Hebel, J. R. (1988). Patient-proxy response comparability on measures of patient health and functional status. Journal of Clinical Epidemiology, 41, 1065-1074.

17. Gifford, J. M., Husain, N., Dinglas, V. D., Colantuoni, E., \& Needham, D. M. (2010). Baseline quality of life before intensive care: A comparison of patient versus proxy responses. Critical Care Medicine, 38, 855-860.

18. Yip, J. Y., Wilber, K. H., Myrtle, R. C., \& Grazman, D. N. (2001). Comparison of older adult subject and proxy responses on the SF-36 health-related quality of life instrument. Aging Mental Health, 5, 136-142.

19. Jensen-Dahm, C., Vogel, A., Waldorff, F. B., \& Waldemar, G. (2012). Discrepancy between self- and proxy-rated pain in Alzheimer's disease: Results from the danish alzheimer intervention study. Journal of the American Geriatrics Society, 60, $1274-1278$.

20. Coast, J., Smith, R., \& Lorgelly, P. (2008). Should the capability approach be applied in health economics? Health Economics, 17, 667-670.

21. Coast, J., Flynn, T., Sutton, E., Al-Janabi, H., Vosper, J., Lavender, S., et al. (2008). Investigating choice experiments for preferences of older people (ICEPOP): Evaluative spaces in health economics. Journal of health services research and policy, 13(Suppl 3), 31-37.

22. Dolan P, R Layard, R Metcalfe (2011) Measuring subjective wellbeing for public policy: Recommendations on measures.

23. Stiglitz J, A Sen, JP Fitoussi (2009) Report by the Commission on the Measurement of Economic Performance and Social Progress.

24. Smith, S. C., Lamping, D. L., Banerjee, S., Harwood, R. H., Foley, B., Smith, P., et al. (2007). Development of a new measure 
of health-related quality of life for people with dementia: DEMQOL. Psychological Medicine, 37, 737-746.

25. Weiner, M. F., Martin-Cook, K., Svetlik, D. A., Saine, K., Foster, B., \& Fontaine, C. S. (2000). The quality of life in late-stage dementia (QUALID) scale. Journal of the American Medical Directors Association, 1, 114-116.

26. Logsdon, R. G., Gibbons, L. E., McCurry, S. M., \& Teri, L. (2002). Assessing quality of life in older adults with cognitive impairment. Psychosomatic Medicine, 64, 510-519.

27. Brazier, J. E., Yang, Y., Tsuchiya, A., \& Rowen, D. L. (2010). A review of studies mapping (or cross walking) non-preference based measures of health to generic preference-based measures. The European journal of health economics, 11, 215-225.

28. Barton, G. R., Sach, T. H., Jenkinson, C., Avery, A. J., Doherty, M., \& Muir, K. R. (2008). Do estimates of cost-utility based on the EQ-5D differ from those based on the mapping of utility scores? Health Quality Life Outcomes, 6, 51.

29. Lawton, M. P. (1997). Assessing quality of life in Alzheimer disease research. Alzheimer Disease and Associated Disorders, 11(Suppl 6), 91-99.

30. Stevens, K. J. (2010). Working with children to develop dimensions for a preference-based, generic, pediatric, health-related quality-of-life measure. Qualitative Health Research, 20, 340-351.

31. Novella, J. L., Jochum, C., Jolly, D., Morrone, I., Ankri, J., Bureau, F., et al. (2001). Agreement between patients' and proxies' reports of quality of life in Alzheimer's disease. Quality of Life Research, 10, 443-452.

32. Cooper C, Mukadam N, Katona C, Lyketsos CG, Blazer D, Ames D, Rabins P, Brodaty H, de Mendonca LC, Livingston G (2012) Systematic Review of the Effectiveness of Pharmacologic Interventions to Improve Quality of Life and Well-being in People With Dementia. Am J Geriatr Psychiatry.

33. Cooper, C., Mukadam, N., Katona, C., Lyketsos, C. G., Ames, D., Rabins, P., et al. (2012). Systematic review of the effectiveness of non-pharmacological interventions to improve quality of life of people with dementia. International Psychogeriatrics, 24, 856-870.

34. Brennan V, S Dixon (2012) A literature review for empirical studies of process utility; ISPOR 17th Annual Internation Meeting.

35. Jones L, Harrington J, Scott S, Davis S, Lord K, Vickerstaff V, Round J, Candy B, Sampson EL (2012) CoMPASs: IOn programme (Care Of Memory Problems in Advanced Stages of dementia: Improving Our Knowledge): protocol for a mixed methods study. BMJ Open 2.

36. Jie, W., Weiss, David J., \& Edwards, Ward. (2010). A descriptive multi-attribute utility model for everyday decisions. Theory and Decision, 68, 101-114.

37. Gigerenzer, G., \& Todd, P. (1999). And the ABC research group: Simple heuristics that make us smart. New York: Oxford University Press.

38. Tversky, A., \& Kahneman, D. (1974). Judgment under uncertainty: Heuristics and biases. Science, 185, 1124-1131.

39. Dolan, P., Lee, H., \& Peasgood, T. (2012). Losing sight of the wood for the trees: Some issues in describing and valuing health, and another possible approach. Pharmacoeconomics, 30, $1035-1049$.

40. Dolan, P. (1999). Whose preferences count? Medical Decision Making, 19, 482-486.

41. Wailoo, A., Tsuchiya, A., \& McCabe, C. (2009). Weighting must wait: incorporating equity concerns into cost-effectiveness analysis may take longer than expected. Pharmacoeconomics, 27, 983-989.

42. Brazier, J., Akehurst, R., Brennan, A., Dolan, P., Claxton, K., McCabe, C., et al. (2005). Should patients have a greater role in valuing health states? Applied Health Economics Health Policy, 4, 201-208. 